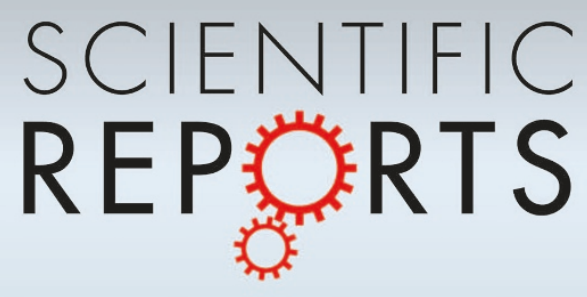

OPEN

SUBJECT AREAS:

SYMBIOSIS

BACTERIAL HOST RESPONSE

Received

19 May 2014

Accepted

2 July 2014

Published

25 July 2014

Correspondence and requests for materials should be addressed to

R.V.M.R. (rita.rio@ mail.wvu.edu)

\title{
Hitchhiking of host biology by beneficial symbionts enhances transmission
}

\author{
Brittany M. Ott, Michael Cruciger, Andrew M. Dacks \& Rita V. M. Rio
}

53 Campus Drive, Department of Biology, West Virginia University, Morgantown, WV 26506.

Transmission plays a key role in the evolution of symbiosis. Mixed mode transmission combines horizontal and vertical mechanisms for symbiont acquisition. However, features that enable mixed transmission are poorly understood. Here, we determine the mechanistic basis for the recruitment of the beneficial bacterium, Aeromonas veronii by the leech, Hirudo verbana. We demonstrate that host mucosal secretions complement imperfect symbiont vertical transmission. First, we show that the $A$. veronii population within secretions originates from the host digestive tract and proliferates synchronously with shedding frequency, demonstrating the coupling of partner biology. Furthermore, leeches are attracted to these castings with oral contact proving sufficient for symbiont transmission. Leech attraction to mucus is not affected by the symbiont state of either the host or mucus, suggesting that $A$. veronii exploits preexisting host behavior and physiological traits. A dual transmission mode, integrating multiple layers of host contributions, may prove evolutionarily advantageous for a wide range of symbioses. Using such a strategy, host infection is ensured, while also providing access to a higher genetic diversity of symbionts. Countless host-associated microbes exhibit mixed mode transmission, supporting the use of the leech symbiosis as a model for enhancing our understanding of the specificity, establishment and persistence of microbiotas.

\footnotetext{
ransmission is instrumental for the colonization and persistence of microbial symbioses ${ }^{1}$. Stable beneficial relations predominantly rely on vertical transmission, often using mechanisms that tap into unique aspects of host reproductive biology. With time, microbial genomes can evolve to complement host biology by eliminating loci involved with extraneous physiological processes. As a trade-off, this genome reduction tends to decrease free-living capabilities and the ability to respond to environmental change (reviewed in ${ }^{2}$ ). In contrast, horizontal transfer of mutualists ${ }^{3}$ requires partner contact and/or infection through the environment. The stochastic nature of partner encounters suggests that a horizontal strategy is consequently less evolutionarily stable $^{4}$, as the horizontal transmission of a genetically diverse symbiont population tends to eliminate congruence with partner phylogenetic trees ${ }^{5}$ (reviewed in ${ }^{4}$ ). A mixed strategy, blending transmission modes, may also occur. While mixed strategies have been previously considered to be transitory in nature, with fitness trade-offs ${ }^{6}$ selecting for the evolutionarily preferred mode ${ }^{7}$, it is possible that the mixed strategy can be the more advantageous scenario particularly if the transmission modes are not exclusive of one another ${ }^{4,8}$. However, the adaptive features that may enable the persistence of an evolutionary favorable mixed strategy are not well understood.

The medicinal leech, Hirudo verbana, uses a mixed strategy for the transmission of a relatively simple microbiota within its digestive tract ${ }^{9}$, yet the mechanistic basis remains unknown. This microbiota is dominated by the Gammaproteobacteria Aeromonas veronii and the Bacteroidia Rikenella-like residents ${ }^{10,11}$, which have been proposed to play a wide range of host fitness enhancing roles (reviewed in ${ }^{12}$ ). A. veronii exhibits diverse lifestyles, as a free-living waterborne microbe and as both a mutualist and a pathogen depending on the host background ${ }^{13-16}$, while members of the Rikenellaceae are found in the guts of a diverse spectrum of animals ${ }^{17-20}$, indicating a high ecological versatility. A. veronii is a pioneer symbiont (i.e. early establishing member of the microbiota) in the crop of $H$. verban $a^{9}$, likely influencing the recruitment of later colonizing symbionts, including Rikenella-like bacteria. The A. veronii symbiont can be vertically transmitted during development within the leech cocoon', yet, only a proportion of juveniles harbor $A$. veronii upon cocoon emergence, and none of the juveniles harbor the Rikenella-like symbiont. Despite this, two-weeks post cocoon emergence, all juvenile leeches harbor both symbiont species ${ }^{9}$, suggesting that some form of horizontal transmission complements imperfect maternal transmission.

Although distinct Aeromonas spp. infect the digestive tracts of medicinal leech species ${ }^{10,11,21}$, displaying tight host-symbiont species specificity, signatures of co-evolution between $A$. veronii and its $H$. verbana host are lacking ${ }^{22}$. This lack of congruence in the phylogenies is in stark contrast to bacterial partners that are vertically transmitted with high fidelity such as the ancient intracellular endosymbionts of some insects (reviewed in ${ }^{2,23}$ ).
} 
High rates of gene flow within $A$. veronii populations have been described $^{24}$, and the presence of several mobile genetic elements, specifically prophages and insertion elements, have been identified within the genome of a leech associated A. veronii isolate, HM21 $1^{25}$, arguing against strict maternal transmission. Such features are lacking in many purely heritable symbionts due to reductive genome evolution within the host (reviewed $\mathrm{in}^{2}$ ). The presence of these genomic features, coupled with incomplete vertical transmission and the absence of deep host-symbiont co-evolution, suggests that symbiont acquisition may also occur from an unknown extrinsic source following cocoon eclosion.

In this study, we identify the mechanistic basis for the mixed transmission strategy of a beneficial symbiont. Specifically, we demonstrate how periodic shedding of leech mucus serves as a naturally occurring substrate for $A$. veronii horizontal transfer to conspecific leech individuals. Leech mucosal secretions consist of glucosaminoglycans produced by globose glands irregularly distributed throughout the epidermis ${ }^{26,27}$ and have been suggested to influence an array of biological functions such as protection from desiccation, respiration and conspecific recognition ${ }^{26,27}$. These are shed by the host at regular intervals, always from the anterior to posterior end, thus providing consistent contact with the digestive tract via the fecal matter. This made mucus a potential candidate to harbor gut symbionts. In support, mucosal secretions provide a substrate for symbiont aggregation and recruitment for many other aquatic animals ${ }^{28-30}$. As such, we examined the potential for mucosal castings to harbor and transmit viable digestive tract symbionts, thus serving as a mechanistic basis for the horizontal component of a mixed transmission strategy.

We report that mucosal secretions harbor proliferating populations of $A$. veronii symbionts. Moreover, leeches are attracted to these secretions, which prove sufficient for horizontal transmission of $A$. veronii. Although symbiont-state did significantly influence leech host exploratory behavior, it did not affect attraction to mucosal castings, suggesting that mutualists are exploiting host behavior and physiological processes that likely evolved for another function prior to the symbiosis. Consistent with this hypothesis, symbiontinfection of mucus did not affect attraction, suggesting that intrinsic properties of the mucus could attract conspecifics, serving to drive host-microbe specificity. These findings suggest that a mixed transmission strategy, exploiting unique facets of host biology, may prove fitness savvy by circumventing trade-offs between transmission modes while also increasing the likelihood of partner encounter for these relatively non-social animals. Remarkably, this strategy may also provide an accessible pool of symbionts with greater genetic flexibility to accommodate environmental changes, while providing alternative lifestyles to $A$. veronii beyond that of mutualism.

\section{Results}

Periodically shed mucosal secretions harbor proliferating microbial symbionts. To determine if leech mucosal secretions (Fig. 1A) house viable $A$. veronii, we assayed the population density of $A$. veronii using quantitative PCR of the single copy gyrB gene locus from mucus samples at two biologically relevant temperatures representing a pond's edge in $H$. verbana's natural geographical distribution ${ }^{31}$, where breeding between leeches typically occurs in summer months $\left(\sim 23^{\circ} \mathrm{C}^{26}\right)$, and a pond's base $\left(\sim 15^{\circ} \mathrm{C}\right)$. At both of these temperatures, $A$. veronii population densities peaked on day 3 and decreased thereafter (Fig. 1B). Although A. veronii harbored within mucus at the two temperatures demonstrated similar growth profiles (Fig. 1B), significant differences in overall density were observed ( $p=0.0086$, one-way ANOVA; $\mathrm{n}=77$ ) with mucus shed at $23^{\circ} \mathrm{C}$ harboring an overall higher symbiont load over the examination period. With time, A. veronii abundance decreased, indicating either an exhaustion of mucosal resources and/or a dispersal of bacteria from the mucus. In addition, symbiont
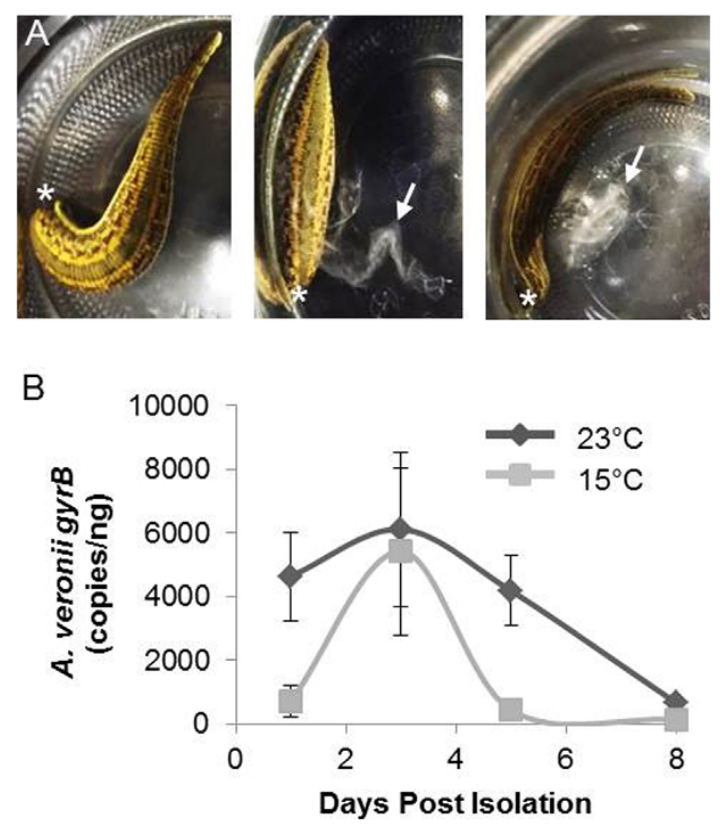

Figure 1 Shed mucosal secretions harbor proliferating microbial symbionts. (A). A leech undergoing the process of mucosal shedding; arrow indicates mucus, asterisk indicates the posterior end of the leech. Mucus is sloughed off from the anterior to posterior end of the leech. (B). A. veronii density within mucus increases from Day 1 to 3 , decreasing thereafter. A. veronii density is measured via qPCR using the single-copy gyrB gene. Significant differences in overall density between the two temperatures examined were observed ( $p=0.0086$, one-way ANOVA; $\mathrm{n}=77$ ).

proliferation peaked approximately when mucus was again secreted by the leech host (Fig. S1), thereby ensuring a continuous supply of seeded mucosal secretions within the environment. Thus, leech gut symbionts are not only viable, but also proliferate in mucus following its secretion in concert with host shedding patterns.

Digestive tract $A$. veronii seed mucosal castings and are sufficient for symbiont transmission. Mucus is shed from the leech posterior (Fig. 1A), enabling the gut symbionts to potentially seed the secretion through the excretory route. This observation implicates the digestive tract as the origin of the mucosal $A$. veronii population. To verify this hypothesis, the castings of leeches that had been cleared of their $A$. veronii gut symbiont via antibiotic treatment (aposymbiotic or APO leeches) were collected and verified to lack A. veronii (Fig. 2A). This observation suggests that digestive tract $A$. veronii are the likely source of the mucosal symbiont population. To further confirm that gut symbionts are transferred to mucus, both $\mathrm{APO}$ and WT leeches were fed a $g f p$-expressing $A$. veronii strain at high $\left(2 \times 10^{4} \mathrm{CFU} / \mathrm{mL}\right)$ and low $\left(2.5 \times 10^{2} \mathrm{CFU} / \mathrm{mL}\right)$ inoculums, which flank the natural $A$. veronii population levels found within the mucus (Fig.1B). Additionally, to determine if gut symbionts persistently seed the mucosal castings of the host, the first casting and a casting 60 days post-feeding (representing $\sim 30^{\text {th }}$ mucosal cast since feeding) were collected and spread on nutrient agar plates and subsequently visualized under fluorescent microscopy (Fig. 2B shows a colony obtained from a first mucosal casting), with their identity confirmed using A. veronii specific gyrB-primers (Fig. S2A). APO and wildtype (WT) leeches secreted their mucosal castings at similar temporal intervals (data not shown). Only oral administration of $g f p$ expressing $A$. veronii at the high inoculum within blood resulted in detectable concentrations within subsequently shed mucus (Fig. 2C; $\mathrm{CFU} / \mathrm{ml}$ grouped across first and $30^{\text {th }}$ castings for APO and WT, $p=$ 0.282 , Mann Whitney $U$-test, $\mathrm{n}=24$ ). With this high oral administration, the percentage of leeches with $A$. veronii symbionts 
A

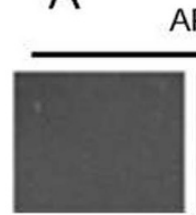

Day 1
APO

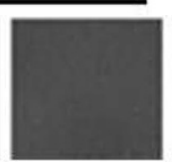

Day 8

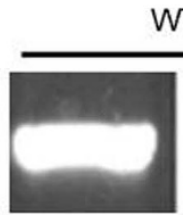

Day 1

WT

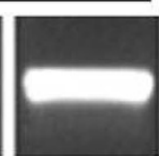

Day 8
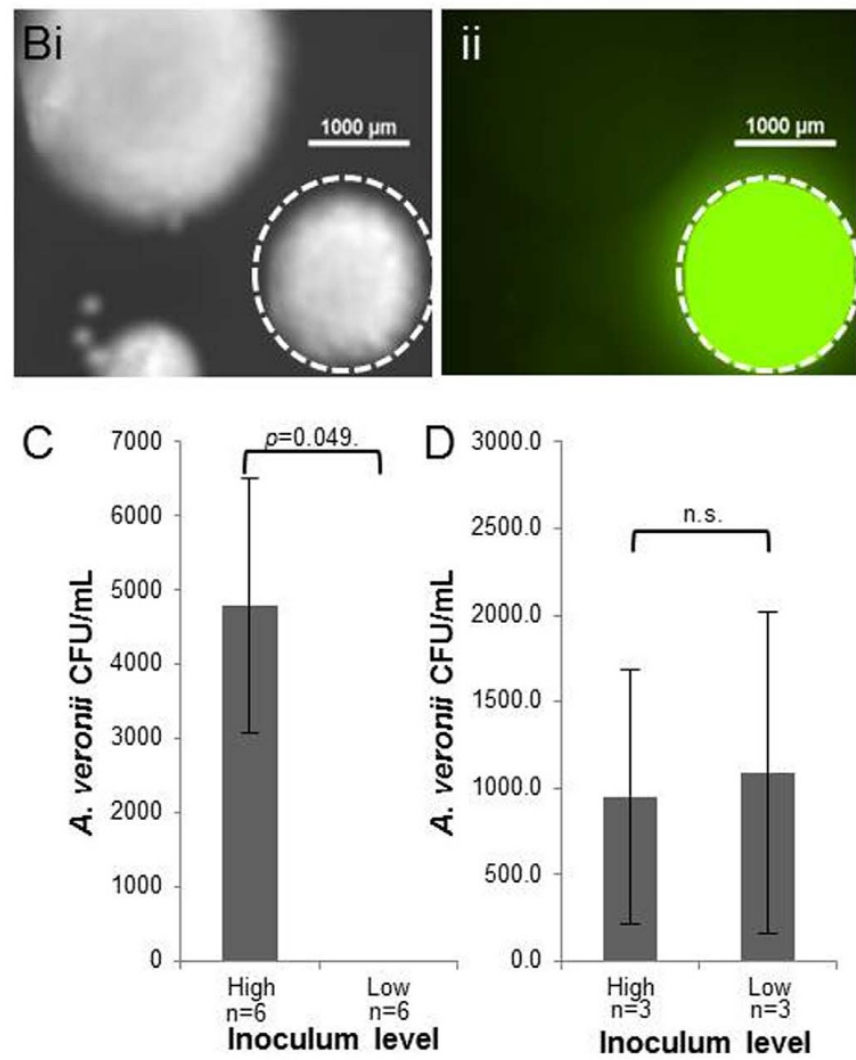

Figure $2 \mid$ Digestive tract $A$. veronii seed mucosal castings, which are sufficient for symbiont transmission. (A). Antibiotic treatment, sufficient to clear gut symbionts (aposymbiotic; APO), also eliminates A. veronii from representative mucosal castings as verified through $A$. veronii specific gyrB PCR. (B). Leeches fed A. veronii possess this symbiont with their first subsequent mucosal shed. i) Brightfield imaging of bacterial colonies from the mucosal secretions of an APO leech fed $g f p$-expressing $A$. veronii mutant HM21S::TN7gfp $\left(2 \times 10^{4} \mathrm{CFU} / \mathrm{mL}\right)$. ii) FITC fluorescent image of $\mathrm{i}$; hatched circles indicate $g f p$-expressing $A$. veronii colony. (C). Fluorescent colony counts within shed mucus of leeches that have been fed $g f p$ -

expressing A. veronii. (D). Fluorescent colony counts within shed mucus of leeches exposed to mucus harboring $g f p$-expressing $A$. veronii. For $C$ and $D$, high and low inoculums were matched to symbiont densities observed in mucus (see Fig. 1B); n.s. = not significant.

present in the first and $\sim 30^{\text {th }}$ casting did not differ (Fig. S2B; $p=$ 0.3865 , Pearson's chi-squared test, $\mathrm{n}=24$ ). Furthermore, $A$. veronii concentrations did not differ between the first and $\sim 30^{\text {th }}$ castings $(p$ $=0.1642$, Student's $t$-test, $\mathrm{n}=11$, data not shown) and there was no effect of leech symbiont state (Fig. S2C; $p=0.7595$, Pearson's chisquared test, $\mathrm{n}=24$ ). These results demonstrate that digestive tract A. veronii establish very rapidly and persistently infect host secretions through time, regardless of leech symbiont state, although the specific route used for colonization of mucus remains unknown.

Although the gut microbiota is capable of seeding the mucosal castings, transmission of these microbes is unlikely to occur through an infected blood meal, as the inoculum loads needed to ensure oral transmission are too high to be encountered. Furthermore, the levels of Aeromonas in the aquatic environments inhabited by leeches are $<1 \mathrm{CFU} / \mathrm{ml}$ (colony forming unit per milliliter of water) ${ }^{32}$. Therefore, it would be highly unlikely for free-living Aeromonas to serve as an inoculum source, as a concentration of $10^{2} \mathrm{CFU} / \mathrm{ml}$ was insufficient for transmission (Fig. 2C). To determine if mucus is capable of horizontally transferring the A. veronii symbiont, APO mucosal secretions were inoculated with high and low densities (as above) of the $g f$-expressing $A$. veronii. These secretions were placed in mesh sacs within individual jars housing an APO leech for seven days. Following this incubation period, leeches were surface-rinsed and moved into new autoclaved jars with sterile LSIO and their first mucosal casts were examined for the presence of $g f p$-expressing $A$. veronii. Oral contact with shed mucus proved sufficient to transmit the A. veronii symbiont at both inoculum levels (Fig. 2D), with no significant difference in the resulting CFU/mL $(p=0.92$, Student's $t$ test, $\mathrm{n}=6$ ) or frequency of individuals containing symbionts within their castings (i.e. 2 of 3 individuals at both concentrations). In multiple invertebrate systems, particularly with regard to vectortransmitted organisms, the natural vector provides a higher efficiency of microbial transmission relative to artificial introduction of the microbe ${ }^{33-40}$. Consistent with this, mucosal exposure at both inoculum levels proved more effective than blood meal $A$. veronii administration at seeding subsequent mucosal casts, supporting mucosal transfer as a natural and efficient method of microbial transmission between leeches.

Leeches display attraction to mucus shed by conspecifics irrespective of host symbiont state. After shedding, mucosal secretions capable of transmitting the $A$. veronii symbiont may float through the water or become caught on debris. Therefore, for mucus to be an effective substrate for transmission, leeches must seek out these castings to enable the transfer of microbes. Here, we sought to determine if leeches are attracted to mucus. Furthermore, because symbiont status affects host behavior in other systems ${ }^{41-43}$, we sought to determine if symbiont-state altered any mucus-oriented behavior. We began by examining the influence of symbiont-state on the baseline behavior of leeches to determine what effects, if any, the antibiotic treatment had on locomotion. APO and WT leeches were placed into an experimental arena (Fig. 3A) and their movements within the arena tracked for ten minutes (Fig. 3B). Heat maps indicating the proportion of time leech subjects spent within an overlaid $10 \times 10$ grid (Fig. 3C) were generated. No significant difference was found between WT and APO leeches in average speed (Fig. S3) ( $p=0.36$, Student's $t$-test, $\mathrm{n}=33$ ), indicating that antibiotics do not affect the ability of the leech to move. In general, leeches were positively thigmotactic (Fig. 3C), preferring contact with the corners and edges of the tank. Interestingly, the two experimental groups did differ in the proportion of time spent away from the edge of the arena (Fig. 3D). APO leeches spent significantly more time in the center of the arena (Fig. $3 \mathrm{E} ; p=$ 0.017 , Student's $t$-test, $\mathrm{n}=33$ ). A willingness to enter the center of an arena is classically associated with "risky" exploratory behavior in vertebrates and invertebrates ${ }^{43-47}$, suggesting that leeches lacking $A$. veronii in their digestive tract produce more exploratory behavior relative to WT leeches.

We hypothesized that the increase in exploratory behavior would raise the likelihood that leeches would encounter the mucosal castings of conspecifics. We therefore examined the behavior of WT and APO leeches in an arena containing two mesh bags: one containing a WT mucus sample and a second empty bag serving as a control (Fig. 4A). The movement of individual leeches within the arena was tracked (Fig. 4B), and the average time distribution of individual leech movement within the experimental arena was determined (Fig. 4C). To investigate leech preference for mucus, we determined the amount of time spent in each of four $2 \times 1$ sub-regions of $10 \times 10$ 

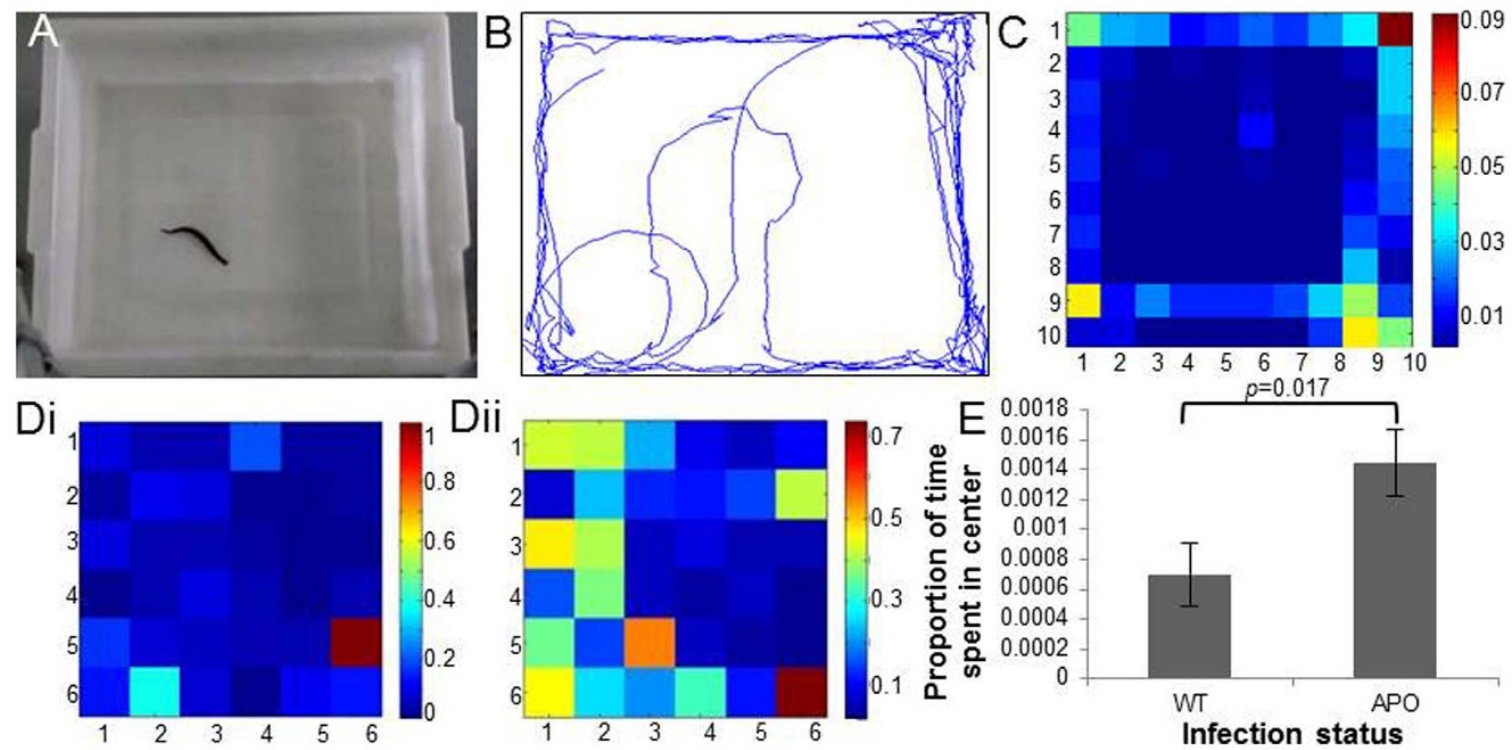

Figure 3 Host symbiont status impacts exploratory behavior. (A). A leech within the general behavior analysis arena. (B). A representative leech movement track obtained during a single recording session. (C). An example of the $10 \times 10$ grid used to observe time spent in each section of the arena, with warmer colors indicating a greater proportion of time spent within that square. (D). The inner $6 \times 6$ squares of the arena depict time spent in the center between i) WT and ii) APO leeches. (E). Baseline behavior assays demonstrate that APO leeches spend significantly more time in the center of the arena than WT ( $p=0.017$, Student's $t$-test, $\mathrm{n}=33$ ), consistent with more exploratory behavior. Error bars indicate 1 Standard Error of the Mean (SEM).

grid (Fig. 4C; overlaid in silico as above). These regions contained the mucus sac, the empty sac, or one of two control regions on the top and bottom sides of the arena (black boxes; Fig. 4C). Leeches in general exhibited a strong preference for the region containing the mucus filled sac relative to the empty sac (Figs. $4 \mathrm{D}$ and $4 \mathrm{E})(p=$ 0.0024 , one-way ANOVA, $\mathrm{n}=37$ ), indicating that leeches are attracted to the mucus of conspecifics. Leeches make frequent, repeated oral contact with the mucus in both our colony tanks and during experiments when the mucus is in the mesh sacs, which is consistent with previous reports that suggest that leeches use mucus for conspecific recognition ${ }^{27}$. However, WT and APO leeches did not differ in the amount of time spent in proximity to the mucus (Fig S4A; $p=0.609$, Student's $t$-test, $\mathrm{n}=36$ ), or in their latency to first investigate the mucus (Fig S4B; $p=0.78$, Student's $t$-test, $\mathrm{n}=23$ ). Therefore, we conclude that shed mucus attracts leeches regardless of host symbiont state, providing an efficient mechanism for transmission of $A$. veronii between leeches.

A. veronii infection of mucus does not impact host attraction. The lack of influence of host symbiont state on attraction to mucus suggested that $A$. veronii is exploiting a preexisting host physiological process most likely derived for another function separate from symbiosis. Alternatively, it is possible that the attraction of leeches to the mucosal castings of conspecifics depends upon the presence of symbionts in the mucus. To determine if the presence of symbionts in the mucus affects host attraction, WT leeches were placed in the behavior arena with two mesh bags; one containing mucus from an APO leech and one containing mucus from a WT leech (Fig. 5A). No significant difference was observed in the time spent in the vicinity of WT versus APO mucus samples (Fig. $5 \mathrm{~B} ; p=0.46$, one-way ANOVA, $\mathrm{n}=18$ ), indicating that an inherent attraction to mucosal secretions occurs between conspecific leeches (Fig. 4C), regardless of $A$. veronii infection. This result is consistent with the suggestion that $A$. veronii likely exploited this preexisting host biological trait.

\section{Discussion}

We have shown that leech mucus can provide a hospitable environment for A. veronii and can serve as a mechanism for horizontal transmission through its ability to attract conspecific leeches. Seed populations of $A$. veronii in the mucus originate from the digestive tract and mucosal administration of $A$. veronii is sufficient for transmission of the symbiont to novel leech hosts. Although oral administration at high inoculum in blood also enabled transmission, the higher efficiency of symbiont transfer via mucus and the improbability that a leech would encounter prey with such high $A$. veronii blood infections, implicate mucus as the natural transmission vehicle. Furthermore, to serve as an effective transmission substrate, leeches would have to actively seek out the mucus and consistent with this we found that leeches are attracted to the mucosal secretions of conspecifics.

Since different Hirudo spp. overlap geographically ${ }^{31}$, but still maintain a high degree of Aeromonas spp. selectivity ${ }^{22}$, we would predict attraction to the mucus of conspecifics over mucus of other leech species. A similar scenario arises with the strict feeding preferences of different species of Anopheles mosquitos that enable the tight coupling with species of the Plasmodium protozoa ${ }^{48-50}$, suggesting that host ecological preferences can have a significant impact on microbial symbioses. A similar host preference for the mucus of conspecifics could drive Aeromonas symbiont selectivity. Alternatively, leech-specific differences in mucosal content may support one species of Aeromonas over another.

As in mice ${ }^{43}$, leeches lacking gut symbionts displayed more exploratory behavior, which we hypothesized would increase their likelihood of encountering the mucus of conspecifics. However, the symbiont status of both the leech and the mucus itself do not have an impact on the time leeches spend near the mucus, indicating that leech attraction to mucus likely preceded the use of mucus for symbiont transmission. However, through this hitchhiking mechanism, the leech is also likely ensured access to an initial and more genetically diverse supply of this necessary symbiont.

The success of the mixed transmission strategy employed by $H$. verbana and $A$. veronii relies on host contribution, specifically an extrinsic substrate synthesized by the host, which is capable of harboring gut symbionts at concentrations that do not occur while $A$. veronii is free-living. Host-generated mucus has been shown to facilitate symbiont recruitment in a number of aquatic animals. For instance, hydra (Hydra vulgaris) maintains a mucus layer, called 

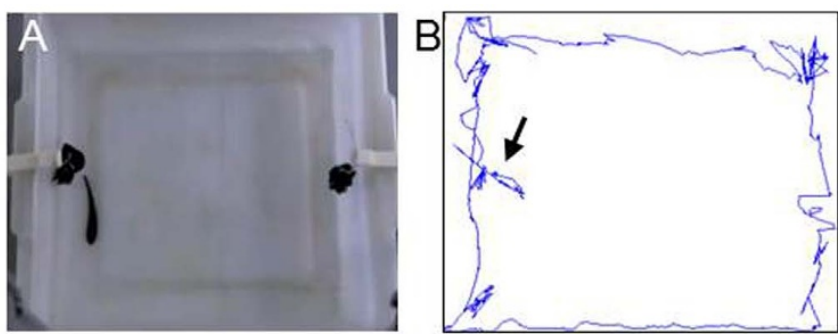

C

Top

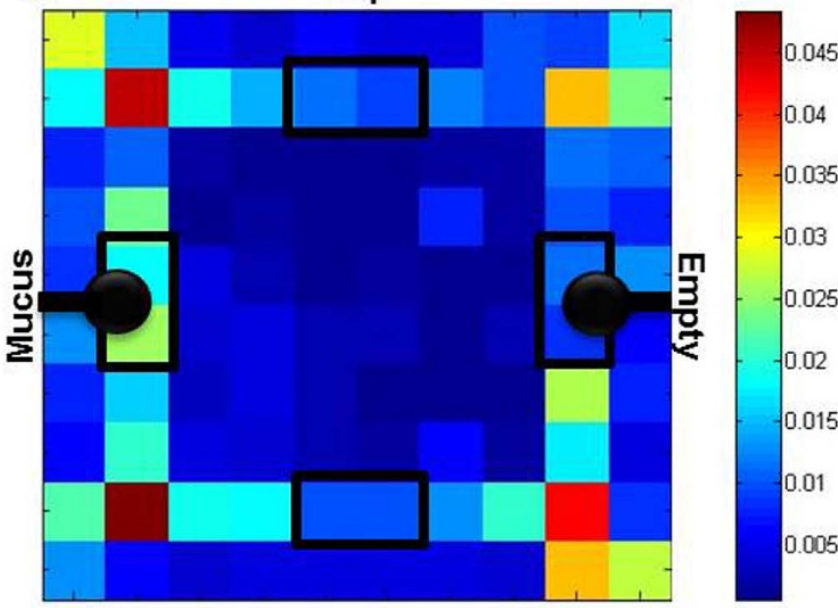

Bottom
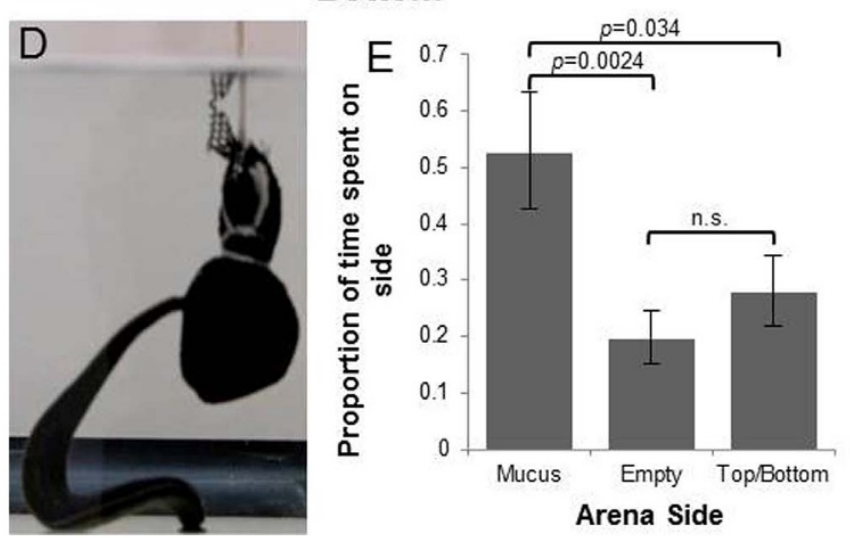

Figure $4 \mid$ Leeches are attracted to mucus shed by conspecifics. (A). A leech within the behavioral analysis arena; mesh bags that are either empty or contain mucus are visible on either side of the arena. (B). A representative leech movement track obtained during a single recording session; arrow indicates location of mesh sac containing mucus. (C). Average time distribution of leech movement in the behavior arena, warmer colors indicate a greater proportion of time spent in that square. Black boxes indicate regions of interest measured for each side of the arena representing the top, bottom, and each side in which the mesh sacs (black circles) are placed. (D). A leech investigating a mucus filled sac. (E).

Leeches spend significantly more time at the mesh sac containing mucus ( $p$ $=0.0024$, one-way ANOVA, $\mathrm{n}=37$ ).

the glycocalyx, where prospective symbionts are recruited and localized during early embyrogenesis ${ }^{51}$. In Euprymna scolopes, the Hawaiian bobtail squid, mucus enables biofilm formation and the aggregation of its light organ symbiont, Vibrio fischeri, prior to the initiation of the symbiosis from a complex milieu of microbes ${ }^{29}$. Horizontal transmission in other model systems requires either direct physical contact, such as sexual transmission, or a vehicle to connect partners (reviewed in ${ }^{1}$ ).

Horizontal transmission involves distinct tradeoffs relative to a free-living lifestyle. For example with horizontal transmission,
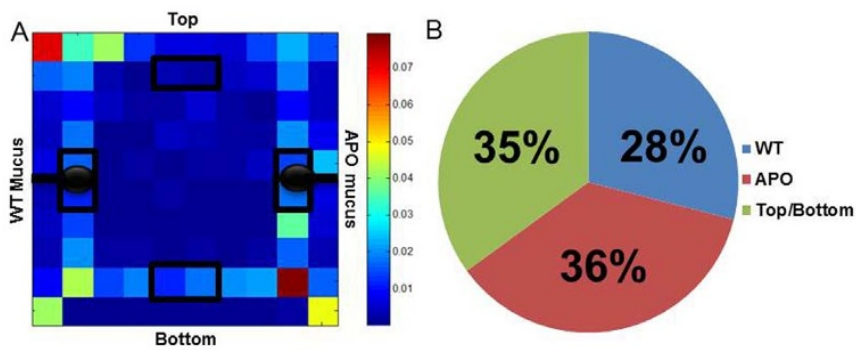

Figure $5 \mid$ Mucus infection does not affect leech attraction. (A). Average time distribution of WT leech movement in the behavior arena in the presence of mucus. Black boxes indicate regions of interest measured for each side of the arena: top, bottom, and each side in which mesh sacs (black circles) contain either WT or APO mucus. (B). There was no significant difference in the time leeches spent near the mesh sac containing either WT or APO mucus ( $p=0.4585$, ANOVA, $\mathrm{n}=18$ ).

energy is typically required for processes such as chemotaxis, motility and adherence to ensure appropriate host encounter and colonization. These costs result in transcriptional profile differences, and potentially investment in structural features (i.e. flagella, pili, etc.) that facilitate horizontal transmission. Consequently, we suggest for a mixed strategy to persist, a host-derived substrate must exist which concentrates symbionts and perhaps even attracts other potential hosts, as in the case of leech mucosal castings, so as to maximize the likelihood of transmission and, theoretically, lessen the cost associated with horizontal transmission.

In recent years, mixed transmission strategies have been recognized as more prevalent than originally anticipated (reviewed in $^{4}$ ). Many symbionts previously thought to be exclusively vertically transmitted may also be transferred horizontally, albeit at low rates $^{5,52-56}$. In fact, symbionts that are exclusively vertically transmitted are rare and currently limited to the obligate mutualists of certain invertebrate taxa (reviewed in ${ }^{1,2}$ ). A variety of circumstances could factor against exclusive vertical transmission. Population bottlenecks during vertical transmission ${ }^{57,58}$ and a lack of available horizontal gene transfer opportunities ${ }^{4}$ result in reduced genetic variation within the symbiont population (reviewed $\mathrm{in}^{2}$ ), limiting any potential adaptation to a changing environment. Importantly, environmental changes to which the host cannot adapt will also result in an evolutionary dead end for its obligate symbiont. Therefore a mixed transmission strategy, which has traditionally been considered transitory in nature ${ }^{7}$, can provide persistent benefits to both host and symbiont, and thereby be positively correlated and selected for. While vertical transmission guarantees that host offspring harbor symbionts, additional complementation via horizontal transmission serves to increase genetic variability of its microbiota, which can enhance the ability of the host to cope with a dynamic environment. However, the degree of genetic variability provided to leech symbionts with mixed relative to vertical modes of transmission is unknown. Furthermore, the microbe also benefits due to lifestyle opportunities other than mutualism.

It is clear that $H$. verbana uses a mixed transmission mode for its pioneer symbiont, $A$. veronii. It remains to be seen however, the degree to which a mixed mode of transmission represents a snapshot in the transition from horizontal to vertical transmission, or if the system represents a stable strategy. Additionally, it remains to be seen how conspecific attraction to mucus may contribute to the high specificity of Aeromonas and leech associations, as Hirudo spp. do geographically overlap ${ }^{31}$. Survival in these secretions could be a mechanism to select for the appropriate symbionts. Similar investigations into the transmission of additional leech symbionts using this mechanism would also be critical in understanding the importance of mucus towards the continuance and stability of the host microbiota. Future work should also explore the role of mixed mode of 
Table 1 | Primer list and amplification settings

\begin{tabular}{|c|c|c|c|}
\hline Primer & Sequence & Amplicon size (bp) & Amplification conditions ${ }^{a}$ \\
\hline Aeromonas veronii gyrase $\mathrm{B}$ (gyrB) & & 514 & $\begin{array}{l}35 \mathrm{cycles} \text { of } 30 \mathrm{sec} \text { at } 95^{\circ} \mathrm{C}, 30 \mathrm{sec} \text { at } \\
53^{\circ} \mathrm{C} \text {, and } 30 \mathrm{sec} \text { at } 72^{\circ} \mathrm{C}\end{array}$ \\
\hline AvgyrB-F & 5'-GCA GAT TGG CGA CAG CAC-3' & & \\
\hline Aeromonas veronii gyrase $B(g y r B)$ q-PCR & & 75 & $\begin{array}{l}35 \text { cycles of } 5 \text { sec at } 98^{\circ} \mathrm{C} \text {, and } 5 \text { sec at } \\
53^{\circ} \mathrm{C}\end{array}$ \\
\hline $\begin{array}{l}\text { QT-AvgyrB For } \\
\text { QT-AvgyrB Rev }\end{array}$ & $\begin{array}{l}\text { 5'-ACT CAC CTC GTT GGC TTC C-3' } \\
\text { 5'-CTT GCT GTA GTC CTC TTT GTC C-3' }\end{array}$ & & \\
\hline
\end{tabular}

transmission in the succession of various members of the microbiota within hosts, as many members of the microbiota are thought to be transmitted as species assemblages ${ }^{4}$. It is possible that a mixed mode of transmission benefits both host and symbiont sufficiently to negate antagonistic selective pressures between vertical and horizontal transmission, particularly if there is considerable host contributions with both modes.

\section{Methods}

(a) Leech husbandry. Wild type (WT) H. verbana leeches were obtained from Leeches USA (Westbury, NY, USA) and maintained in sterilized, Leech Strength Instant Ocean $\mathrm{H}_{2} \mathrm{O}(0.004 \%$ IO, LSIO) in the Department of Biology at West Virginia University at either $15^{\circ} \mathrm{C}$ or $23^{\circ} \mathrm{C}$. Leeches were maintained on defibrinated bovine blood (Hemostat, CA). Aposymbiotic (APO) leeches, in this study referred to leeches lacking their $A$. veronii gut symbiont, were generated through the administration of at least one blood meal containing an antibiotic cocktail of $15 \mu \mathrm{g} / \mathrm{mL}$ of kanamycin $(\mathrm{Km})$ and $10 \mu \mathrm{g} / \mathrm{mL}$ of rifaximin (Rif).

(b) Mucosal secretion sampling. Tanks were cleaned to remove all mucus and fresh LSIO added (denoted as Day 0). Mucosal samples gathered the following day were considered $1 \mathrm{~d}$ old or were aged at the temperatures at which they were extracted for 3,5 or $8 \mathrm{~d}$ within LSIO. All samples were snap frozen at $-80^{\circ} \mathrm{C}$ until further processing. To determine host mucosal secretion rate, individual leeches were maintained in LSIO, with daily examination for the shed castings.

(c) Symbiont population dynamics. The population dynamics of $A$. veronii symbionts within host mucus at $1,3,5$ and $8 \mathrm{~d}$ following secretion were determined by utilizing real-time quantitative PCR (q-PCR). DNA isolation from mucus was performed following a Holmes-Bonner protocol ${ }^{59}$. Analyses were performed in an iCycler iQ Real-Time PCR Detection System (Bio-Rad, Hercules, CA, USA) using BioRad SsoFast EvaGreen Supermix, $10 \mathrm{mM}$ of primers (QT-AvgyrB For and QT-AvgyrB Rev q-PCR for the amplification of $A$. veronii (Table 1$)$ ), and $2 \mu \mathrm{L}$ of DNA template as described ${ }^{9}$. Quantification of the amplicons relative to standard curves was performed using Bio-Rad CFX Manager software v 2.0. The respective DNA concentrations (ng/ ul) of samples were used for the normalization of copy numbers. All assays were performed in triplicate and replicates were averaged for each sample.

(d) Behavioral testing. Behavioral assays, comparing baseline locomotion between WT and APO leeches, were performed by placing leech individuals within tanks $(44.5 \mathrm{~cm} \times 36.2 \mathrm{~cm})$ filled with $10 \mathrm{~L}$ of sterile LSIO. Fresh LSIO and a new sterilized tank were provided for each leech during behavioral testing. Behavior was recorded for 10 min with a Casio Exilim 60 FPS Digital Camera (Ex-F1) and video footage processed using VirtualDub v.1.9.11 (Avery Lee, Informer Technologies). Leech movement was tracked using CTrax v.0.3.14 ${ }^{60}$ and analyzed using custom MatLab v.8.1.0.604 scripts (MathWork, Natick, MA, USA). The tanks were divided into $10 \times$ 10 grids in silico and heat maps depicting the proportion of time spent in each grid unit of the experimental tank were generated. Experiments were performed in the same two-hour time frame every day to eliminate any temporal bias and all assays were performed and analyzed with the observer blind to symbiont status of the individual leeches and the presence/absence of symbionts within mucus castings.

Three behavioral assays were performed. The goal of the first assay was to determine if symbiont status (i.e. WT or APO) affected baseline locomotion of leeches. The average speed and relative proportion of time spent by leeches in the empty behavioral arena was calculated over the ten-minute testing period. The leeches were then rested for one week. The goal of the second behavioral assay was to determine if leeches were attracted to mucosal secretions and if there were any differences in level of attraction based on symbiont-state. Mesh sacs (\#9013, Seattle Fabrics Inc., Seattle, WA), either empty or containing WT mucus, were suspended from opposite sides of the behavioral arena and the behavior of WT or APO leeches observed over a 10 minute period. These sacs served the purpose of containment, to control the location of mucus in our experiments, and to facilitate visualization as the mucus is translucent. It should be noted that this mesh is porous, enabling leeches to still make physical contact. The sides containing the empty or mucus mesh balls were switched between individual trials to eliminate any potential side bias. The objective of the third behavioral assay was to determine if the presence of symbionts in the mucosal castings affected attraction to the mucus. WT leeches were placed in the behavioral arena with mesh sacs containing mucus from either WT or APO leeches. The sides containing the WT or APO mucus mesh balls were switched between leech individuals to eliminate any potential side bias.

(e) Transmission assays. To assess whether symbionts introduced into the digestive tract of $H$. verbana were capable of establishing within mucosal secretions, APO and WT leeches were fed $5 \mathrm{~mL}$ of defibrinated bovine blood supplemented with either a low $(250 \mathrm{CFU} / \mathrm{mL})$ or high $(20,000 \mathrm{CFU} / \mathrm{mL})$ inoculum of a constitutively $g f p$ expressing A. veronii mutant, HM21S::Tn7gfp (kindly provided by the Graflab, Univ. of Connecticut). To assess both $A$. veronii transmission and stability within mucus through time, the first mucus and a mucus shed approximately sixty days following feeding were obtained and examined for the presence of HM21S::Tn7gfp by homogenizing and spreading the samples on selective nutrient agar plates ( $\mathrm{LB}+\mathrm{Km}$ $(100 \mu \mathrm{g} / \mathrm{mL})+$ Streptomycin $(\mathrm{Str} ; 100 \mu \mathrm{g} / \mathrm{mL}))$ incubated overnight at $30^{\circ} \mathrm{C}$. Plates were then screened for $g f p$-expressing colonies using a Nikon Eclipse $\mathrm{Ti}^{1}$ inverted fluorescence microscope and images were taken using NIS Elements Imaging Analysis Software. Single colonies were confirmed to be $A$. veronii through PCR using gyrB primers (Table 1) and sequencing of amplicons.

A second form of transmission assay was used to assess whether mucus can serve as natural mechanism for symbiont acquisition. To accomplish this, APO leeches were placed in jars containing mucosal castings in autoclaved mesh bags (see above) for seven days. The mucosal samples used in these studies were isolated from APO leeches, verified free of $A$. veronii through PCR, and were subsequently inoculated with either high or low HM21S::Tn7gfp concentrations (see above). Following the exposure period, leeches were rinsed in sterile LSIO to ensure removal of any residual mucus. Leeches were subsequently placed in autoclaved jars with sterile LSIO and their first mucosal casts were examined for the presence of HM21S::Tn7gfp as described above.

(f) Statistical analyses. The data were analyzed using JMP 10 (SAS Institute Inc., Cary, NC, USA) software. Statistically significant differences in symbiont densities within secreted mucus through time were determined by performing F-tests, followed by the appropriate $t$-test (Mann-Whitney or Student's). To compare frequency of $A$. veronii colonies expressing $g f p$ within mucus following oral administration, Pearson's chisquared tests were used. ANOVAs were performed to determine if leeches demonstrated side preference in experimental arenas during behavioral assays. Mean APO and WT leech speed ( $\mathrm{mm}$ traveled/sec) was determined and compared using a two-tailed Student's $t$-test. Lastly, the exploratory behavior of APO and WT individuals was measured by calculating total time spent in the inner $6 \times 6$ matrix (denoting the center of the arena) and compared between the two groups with a Student's $t$-test.

1. Bright, M. \& Bulgheresi, S. A complex journey: transmission of microbial symbionts. Nature Rev. Microbiol. 8, 218-230 (2010).

2. Moran, N. A., McCutcheon, J. P. \& Nakabachi, A. Genomics and evolution of heritable bacterial symbionts. Annu. Rev. Genet. 42, 165-190 (2008).

3. Sachs, J. L., Skophammer, R. G. \& Regus, J. U. Evolutionary transitions in bacterial symbiosis. Proc. Natl. Acad. Sci. U.S.A. 108 Suppl 2, 10800-10807 (2011).

4. Ebert, D. The Epidemiology and Evolution of Symbionts with Mixed-Mode Transmission. Annu. Rev. Ecol. Evol. Syst. 44, 623-643 (2013).

5. Brandvain, Y., Goodnight, C. \& Wade, M. J. Horizontal transmission rapidly erodes disequilibria between organelle and symbiont genomes. Genetics 189, 397-404 (2011).

6. Sachs, J. L., Russell, J. E. \& Hollowell, A. C. Evolutionary instability of symbiotic function in Bradyrhizobium japonicum. PloS One 6, e26370 (2011).

7. Ewald, P. W. Transmission modes and evolution of the parasitism-mutualism continuum. Ann. N.Y. Acad. Sci. 503, 295-306 (1987).

8. van den Bosch, F., Fraaije, B. A., van den Berg, F. \& Shaw, M. W. Evolutionary bistability in pathogen transmission mode. Proc. R. Soc. B. 277, 1735-1742 (2010).

9. Rio, R. V. et al. Symbiont succession during embryonic development of the European medicinal leech, Hirudo verbana. Appl. Environ. Microbiol. 75, 6890-6895 (2009). 
10. Worthen, P. L., Gode, C. J. \& Graf, J. Culture-independent characterization of the digestive-tract microbiota of the medicinal leech reveals a tripartite symbiosis. Appl. Environ. Microbiol. 72, 4775-4781 (2006).

11. Laufer, A. S., Siddall, M. E. \& Graf, J. Characterization of the digestive-tract microbiota of Hirudo orientalis, a european medicinal leech. Appl. Environ. Microbiol. 74, 6151-6154 (2008).

12. Graf, J., Kikuchi, Y. \& Rio, R. V. Leeches and their microbiota: naturally simple symbiosis models. Trends Microbiol. 14, 365-371 (2006).

13. Graf, J. Symbiosis of Aeromonas veronii biovar sobria and Hirudo medicinalis, the medicinal leech: a novel model for digestive tract associations. Infect. Immun. 67, 1-7 (1999).

14. Janda, J. M. \& Abbott, S. L. Evolving concepts regarding the genus Aeromonas: An expanding panorama of species, disease presentations, and unanswered questions. Clin. Infect. Dis. 27, 332-344 (1998).

15. Bates, J. M. et al. Distinct signals from the microbiota promote different aspects of zebrafish gut differentiation. Dev. Biol. 297, 374-386 (2006).

16. Hu, M. et al. Identity and virulence properties of Aeromonas isolates from diseased fish, healthy controls and water environment in China. Lett. Appl. Microbiol. 55, 224-233 (2012).

17. Kaneuchi, C. \& Mitsuoka, T. Bacteroides microfusus, a new species from the intestine of calves, chickens and Japanese quails. Int. J. Syst. Bacteriol. 28, 478-481 (1978).

18. Ley, R. E. et al. Obesity alters gut microbial ecology. Proc. Natl. Acad. Sci. U.S.A. 102, 11070-11075 (2005)

19. Eckburg, P. B. et al. Diversity of the human intestinal microbial flora. Science 308, 1635-1638 (2005).

20. Rautio, M. et al. Bacteriology of histopathologically defined appendicitis in children. Pediatr. Infect. Dis. J. 19, 1078-1083 (2000).

21. Siddall, M. E., Worthen, P. L., Johnson, M. \& Graf, J. Novel role for Aeromonas jandaei as a digestive tract symbiont of the North American medicinal leech. Appl. Environ. Microbiol. 73, 655-658 (2007).

22. Siddall, M. E. et al. Bacterial symbiont and salivary peptide evolution in the context of leech phylogeny. Parasitology 138, 1815-1827 (2011)

23. Wernegreen, J. J. Genome evolution in the bacterial endosymbionts of insects. Nat. Rev. Genet. 3, 850-861 (2002).

24. Silver, A. C. et al. Complex evolutionary history of the Aeromonas veronii group revealed by host interaction and DNA sequence data. PloS One 6, e16751 (2011).

25. Bomar, L. et al. Draft Genome Sequence of Aeromonas veronii Hm21, a Symbiotic Isolate from the Medicinal Leech Digestive Tract. Genome Announc. 1 (2013).

26. Sawyer, R. T. Leech biology and behavior. (Clarendon Press, 1986).

27. Michalsen, A., Roth, M. \& Dobos, G. Medicinal leech therapy. (Thieme Medical Publishers, 2007)

28. Lema, K. A., Willis, B. L. \& Bourne, D. G. Corals form characteristic associations with symbiotic nitrogen-fixing bacteria. Appl. Environ. Microbiol. 78, 3136-3144 (2012)

29. Nyholm, S. V. et al. Roles of Vibrio fischeri and nonsymbiotic bacteria in the dynamics of mucus secretion during symbiont colonization of the Euprymna scolopes light organ. Appl. Environ. Microbiol. 68, 5113-5122 (2002).

30. Rohwer, F., Seguritan, V., Azam, F. \& Knowlton, N. Diversity and distribution of coral-associated bacteria. Mar. Ecol. Prog. Ser. 243, 1-10 (2002).

31. Utevsky, S. et al. Distribution and status of medicinal leeches (genus Hirudo) in the Western Palaearctic: anthropogenic, ecological, or historical effects? Aquat. Conserv. 20, 198-210 (2010)

32. Holmes, P., Niccolls, L. M. \& Sartory, D. P. in The Genus Aeromonas (eds Austin, B., Altwegg, M., Gosling, P. J. \& Joseph, S.) 127-150 (John Wiley \& Sons Ltd., 1996).

33. Belkaid, Y. et al. Development of a natural model of cutaneous leishmaniasis: powerful effects of vector saliva and saliva preexposure on the long-term outcome of Leishmania major infection in the mouse ear dermis. J. Exp. Med. 188, 1941-1953 (1998)

34. Caljon, G. et al. Tsetse fly saliva accelerates the onset of Trypanosoma brucei infection in a mouse model associated with a reduced host inflammatory response. Infect. Immun. 74, 6324-6330 (2006).

35. Marchal, C. et al. Antialarmin effect of tick saliva during the transmission of Lyme disease. Infect. Immun. 79, 774-785 (2011).

36. Norsworthy, N. B. et al. Sand fly saliva enhances Leishmania amazonensis infection by modulating interleukin-10 production. Infect. Immun. $\mathbf{7 2}$ 1240-1247 (2004)

37. Samuelson, J., Lerner, E., Tesh, R. \& Titus, R. A mouse model of Leishmania braziliensis braziliensis infection produced by coinjection with sand fly saliva. J. Exp. Med. 173, 49-54 (1991).

38. Spence, P. J. et al. Vector transmission regulates immune control of Plasmodium virulence. Nature 498, 228-231 (2013).

39. Theodos, C. M., Ribeiro, J. M. \& Titus, R. G. Analysis of enhancing effect of sand fly saliva on Leishmania infection in mice. Infect. Immun. 59, 1592-1598 (1991).

40. Titus, R. G. \& Ribeiro, J. M. Salivary gland lysates from the sand fly Lutzomyia longipalpis enhance Leishmania infectivity. Science 239, 1306-1308 (1988).

41. Cryan, J. F. \& O'Mahony, S. M. The microbiome-gut-brain axis: from bowel to behavior. Neurogastroenterol. Motil. 23, 187-192 (2011).

42. Desbonnet, L. et al. The probiotic Bifidobacteria infantis: An assessment of potential antidepressant properties in the rat. J. Psychiatr. Res. 43, 164-174 (2008).
43. Heijtz, R. D. et al. Normal gut microbiota modulates brain development and behavior. Proc. Natl. Acad. Sci. U.S.A. 108, 3047-3052 (2011).

44. Bilbo, S. D. et al. Neonatal infection induces memory impairments following an immune challenge in adulthood. Behav. Neurosci. 119, 293-301 (2005).

45. Crawley, J. N. Behavioral phenotyping strategies for mutant mice. Neuron $\mathbf{5 7}$ 809-818 (2008).

46. Goehler, L. E. et al. Campylobacter jejuni infection increases anxiety-like behavior in the holeboard: possible anatomical substrates for viscerosensory modulation of exploratory behavior. Brain Behav. Immun. 22, 354-366 (2008).

47. Sullivan, R. et al. The International Society for Developmental Psychobiology annual meeting symposium: Impact of early life experiences on brain and behavioral development. Dev. Psychobiol. 48, 583-602 (2006).

48. van den Broek, I. V. F. \& den Otter, C. J. Olfactory sensitivities of mosquitoes with different host preferences (Anopheles gambiae s.s., An. arabiensis, An. quadriannulatus, An. m. atroparvus) to synthetic host odours. J. Insect Physiol. 45, 1001-1010 (1999).

49. Wekesa, J. W., Copeland, R. S. \& Mwangi, R. W. Effect of Plasmodium falciparum on blood feeding behavior of naturally infected Anopheles mosquitoes in western Kenya. Am. J. Trop. Med. Hyg. 47, 484-488 (1992).

50. White, G. B. Anopheles gambiae complex and disease transmission in Africa. Trans. R. Soc. Trop. Med. Hyg. 68, 278-301 (1974).

51. Fraune, S. et al. In an early branching metazoan, bacterial colonization of the embryo is controlled by maternal antimicrobial peptides. Proc. Natl. Acad. Sci. U.S.A. 107, 18067-18072 (2010).

52. Dunn, A. M. \& Smith, J. E. Microsporidian life cycles and diversity: the relationship between virulence and transmission. Microbes Infect. 3, 381-388 (2001).

53. Huigens, M. E. et al. Infectious parthenogenesis. Nature 405, 178-179 (2000).

54. Moran, N. A. \& Dunbar, H. E. Sexual acquisition of beneficial symbionts in aphids. Proc. Natl. Acad. Sci. U.S.A. 103, 12803-12806 (2006).

55. Snyder, A. K., McMillen, C. M., Wallenhorst, P. \& Rio, R. V. The phylogeny of Sodalis-like symbionts as reconstructed using surface-encoding loci. FEMS Microbiol. Lett. 317, 143-151 (2011).

56. Werren, J. H. \& van den Assem, J. Experimental analysis of a paternally inherited extrachromosomal factor. Genetics 114, 217-233 (1986).

57. Mira, A. \& Moran, N. A. Estimating population size and transmission bottlenecks in maternally transmitted endosymbiotic bacteria. Microb. Ecol. 44, 137-143 (2002).

58. Rio, R. V., Wu, Y. N., Filardo, G. \& Aksoy, S. Dynamics of multiple symbiont density regulation during host development: tsetse fly and its microbial flora. Proc. Biol. Sci. 273, 805-814 (2006)

59. Holmes, D. S. \& Bonner, J. Preparation, molecular weight, base composition, and secondary structure of giant nuclear ribonucleic acid. Biochemistry 12, 2330-2338 (1973).

60. Branson, K. et al. High-throughput ethomics in large groups of Drosophila. Nature methods 6, 451-457 (2009).

\section{Acknowledgments}

We thank Dr. Joerg Graf for kindly providing HM21S::Tn7gfp, Dr. Josh Martin for providing custom MatLab scripts, Dr. Jim Belanger for use of video equipment and Grace Altimus for technical assistance. We thank Dr. Penny Dacks for reviewing the manuscript and providing useful feedback. We gratefully acknowledge the funding support of NSF IOS-1025274 and NSF DBI-1156627.

\section{Author contributions}

Conceived and designed the experiments: B.O., A.D. and R.R. Performed the experiments: B.O. and M.C. Analyzed data: B.O., M.C., A.D. and R.R. Contributed the reagents/material/ analysis tools: A.D. and R.R. Wrote the paper: B.O., M.C., A.D. and R.R. All authors reviewed the paper.

\section{Additional information}

Supplementary information accompanies this paper at http://www.nature.com/ scientificreports

Competing financial interests: The authors declare no competing financial interests.

How to cite this article: Ott, B.M., Cruciger, M., Dacks, A.M. \& Rio, R.V.M. Hitchhiking of host biology by beneficial symbionts enhances transmission. Sci. Rep. 4, 5825; DOI:10.1038/ srep05825 (2014)

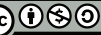

This work is licensed under a Creative Commons Attribution-NonCommercialShareAlike 4.0 International License. The images or other third party material in this article are included in the article's Creative Commons license, unless indicated otherwise in the credit line; if the material is not included under the Creative Commons license, users will need to obtain permission from the license holder in order to reproduce the material. To view a copy of this license, visit http:// creativecommons.org/licenses/by-nc-sa/4.0/ 